Pacific Journal of Mathematics

GAUGE GROUPS AND CLASSIFICATION OF BUNDLES WITH 


\title{
GAUGE GROUPS AND CLASSIFICATION OF BUNDLES WITH SIMPLE STRUCTURAL GROUP
}

\author{
W. D. Curtis and F. R. Miller
}

Suppose $\pi_{i} i=1,2$ are principal $K$-bundles which are $C^{r}$-isomorphic in the sense that there exists a $K$-equivariant $C^{r}$-diffeomorphism $f: \mathscr{P}_{1} \rightarrow \mathscr{P}_{2}$. If $h$ belongs to the gauge group $H_{2}$ of $\mathscr{P}_{2}$ then $h \circ f$ lies in $H_{1}$ and we have a group isomorphism $H_{2} \rightarrow H_{1}$ which is $C^{\infty}$. It is the purpose of this paper to investigate the converse in the case where $K$ is a simple Lie group. (If $K$ is abelian the gauge group of every $K$ bundle over $X$ is $C^{r}(X, K)$ so there is no hope of a converse. However for simple groups the situation is much better).

0. Introduction. Let $K$ be a compact connected Lie group with Lie algebra $\mathscr{K}$. Let $\pi: \mathscr{P} \rightarrow X$ be a principal $K$-bundle of class $C^{\infty}$ where $X$ is a compact, connected $C^{\infty}$-manifold.

Throughout this paper $r$ will be a positive integer which is chosen at this time and remains unchanged from here on.

We denote by $H$ the subgroup of $C^{r}(\mathscr{P}, K)$ consisting of all those $h$ for which $h(p k)=k^{-1} h(p) k$ for all $p$ in $\mathscr{P}$ and $k \in K$. $H$ is naturally isomorphic to the group of all $C^{r}$-bundle automorphisms of $\mathscr{P}$ which cover the identity on $X[1,2]$. The group $H$ will be called the gauge group of $\pi$ the terminology being motivated by current usage in theoretical physics. $C^{r}(\mathscr{P}, K)$ is a Banach Lie group and $H$ is a sub-manifold and so $H$ is a Banach Lie group [2]. The Lie algebra of $H$ can be identified as $\mathscr{H}=\left\{h: \mathscr{P} \rightarrow \mathscr{K} \mid h\right.$ is $C^{r}$ and $h(p k)=A d\left(k^{-1}\right) h(p)$ for $p \in \mathscr{P}, k \in K\}$.

The bracket in $\mathscr{H}$ and the exponential map exp: $\mathscr{H} \rightarrow H$ are the natural pointwise operations.

1. Ideals in $\mathscr{H}$. Suppose $\mathscr{I} \subset \mathscr{H}$ is an ideal. For $p \in \mathscr{P}$ $e_{p}: \mathscr{H} \rightarrow \mathscr{K}$ is defined by $e_{p}(h)=h(p)$ for $h \in \mathscr{H} . \quad e_{p}$ is a Lie algebra epimorphism so $e_{p}(\mathscr{I})$ is an ideal in $\mathscr{K}$.

Lemma 1.1. If $p \in \mathscr{P}$ and $k \in K$ then $e_{p}(\mathscr{I})=e_{p k}(\mathscr{I})$.

Proof. $\quad e_{p k}(h)=h(p k)=A d\left(k^{-1}\right) h(p)=A d\left(k^{-1}\right) e_{p}(h)$. Thus $e_{p k}(\mathscr{I})=A d\left(k^{-1}\right) e_{p}(\mathscr{I})$. But $e_{p}(\mathscr{I})$ is an ideal in $\mathscr{K}$ so $A d\left(k^{-1}\right) e_{p}(\mathscr{I})=$ $e_{p}(\mathscr{I})$.

Definition 1.2. If $x \in X$ let $\mathscr{K}_{x}=e_{p}(\mathscr{I})$ where $p \in \pi^{-1}(x)$. 
DefinItION 1.3 . If $\mathscr{I}$ is an ideal in $\mathscr{H}$ we say $\mathscr{I}$ has property $s$ if $[\mathscr{I}, \mathscr{H}]=\mathscr{I}$.

We recall that $[\mathscr{I}, \mathscr{H}]$ is the Lie subalgebra of $\mathscr{H}$ generated by all elements of the form $[a, b]$ where $a \in \mathscr{I}, b \in \mathscr{H}$. $[\mathscr{I}, \mathscr{H}]$ consists exactly of all finite sums $\Sigma_{i}\left[a_{t}, b_{t}\right], a_{t} \in \mathscr{I}, b_{i} \in \mathscr{H}$.

We denote by $\mathscr{F}(X)$ the algebra of $C^{r}$, real valued functions on $X$. $\mathscr{H}$ is a module over $\mathscr{F}(X)$ for if $f \in \mathscr{F}(X)$ and $h \in \mathscr{H}$ define $f h: \mathscr{P} \rightarrow \mathscr{K}$ by $(f h)(p)=f(\pi(p)) h(p)$. One easily sees $f h$ lies in $\mathscr{H}$ so we have a module.

LEMmA 1.4. If the ideal $\mathscr{I} \subset \mathscr{H}$ has property $s$ then $\mathscr{I}$ is a $\mathscr{F}(X)$ submodule of $\mathscr{H}$.

Proof. Let $h \in \mathscr{I}, \phi \in \mathscr{F}(X)$. We show $\phi h \in \mathscr{I} . \quad \mathscr{I}$ has property $s$ so we may write $h=\Sigma_{t}\left[h_{\imath}, f_{l}\right]$ where $h_{i} \in \mathscr{I}$ and $f_{t} \in \mathscr{H}$. Then $\phi h=$ $\Sigma_{l} \phi\left[h_{i}, f_{l}\right]=\Sigma_{l}\left[h_{i}, \phi f_{l}\right] \in \mathscr{I}$ where we used the pointwise nature of the bracket to get the last equation.

LEMMA 1.5. If $\mathscr{H}_{1}$ and $\mathscr{H}_{2}$ correspond to bundles $\pi_{1}$ and $\pi_{2}$ and $\psi: \mathscr{H}_{1} \rightarrow \mathscr{H}_{2}$ is a Lie algebra isomorphism then if $\mathscr{I}$ has property s in $\mathscr{H}_{1}$ then $\psi(\mathscr{I})$ has property $s$ in $\mathscr{H}_{2}$.

Before proving the final lemma of this section we make a preliminary construction. Suppose $U$ is open in $X$ and $\xi$ is a section of $\pi$ over $U$. Suppose $h \in \mathscr{H}$ and $h$ has support in $\pi^{-1}(U)$. Define $\bar{h}: X \rightarrow \mathscr{K}$ by,

$$
\bar{h}(x)= \begin{cases}h(\xi(x)) & x \in U \\ 0 & x \notin U .\end{cases}
$$

$\bar{h} \in C^{r}(X, \mathscr{K})$ has support in $U$. Conversely if we start with $\bar{h}: X \rightarrow \mathscr{K}$ having support in $U$ we can define $h \in \mathscr{H}$ as follows. There is a unique $C^{\infty}$-map $\theta: \pi^{-1}(U) \rightarrow \mathscr{K}$ such that $\xi(\pi(p)) \theta(p)=p$ for $p \in \pi^{-1}(U)$. We define

$$
h(p)= \begin{cases}\operatorname{Ad}\left(\theta(p)^{-1}\right) \bar{h}(\pi(p)) & p \in \pi^{-1}(U) \\ 0 & p \notin \pi^{-1}(U) .\end{cases}
$$

It is easily checked that $h \in \mathscr{H}$. 
If $x_{0} \in X$ we have:

$$
\begin{aligned}
& H_{x_{0}}=\left\{f \in H \mid f(p)=e \text { for all } p \in \pi^{-1}\left(x_{0}\right)\right\} . \\
& \mathscr{H}_{x_{0}}=\left\{h \in \mathscr{H} \mid h(p)=0 \text { for all } p \in \pi^{-1}\left(x_{0}\right)\right\} .
\end{aligned}
$$

Lemma 1.6. Assume $\mathscr{K}$ is semisimple. Then $\mathscr{H}_{x_{0}}$ has property $s$.

Proof. Let $\left(\phi_{i}\right)_{i}$ be a finite partition of unity on $X$ subordinate to an open cover $\left(U_{l}\right)_{i}$ such that $\pi$ is trivial over each $U_{l}$. Then if $h \in \mathscr{H}_{x_{0}}$ we have $h=\Sigma_{i} \phi_{i} h$ and each $\phi_{i} h \in \mathscr{H}_{x_{0}}$. Therefore the problem is reduced to proving the following: If $U \subset X$ is open such that $\pi$ has a local section $\xi$ defined on $U$ and if $h \in \mathscr{H}_{x_{0}}$ has support in $\pi^{-1}(U)$ then $h$ can be written as $h=\Sigma_{\nu}\left[g_{\nu}, \phi_{\nu}\right]$ where $g_{\nu} \in \mathscr{H}_{x_{0}}, \phi_{\nu} \in \mathscr{H}$.

Let $\bar{h}: X \rightarrow \mathscr{K}$ correspond to $h$ using the section $\xi$ as above. Let $\left(E_{l}\right)_{i}$ be a basis for $\mathscr{K}$. Write $\bar{h}=\sum_{l} \bar{h}^{\prime} E_{l}$ where $\bar{h}^{\prime}$ are real valued. Since $\mathscr{K}$ is semisimple we may write $E_{l}=\sum_{j}\left[F_{i j}, G_{i j}\right]$ where $F_{i j}, G_{i j}$ are in $\mathscr{K}$. Therefore $h=\sum_{i, j} \bar{h}^{i}\left[F_{i j}, G_{i j}\right]=\sum_{i, j}\left[\bar{h}^{i} F_{i j}, G_{i j}\right]=\sum_{\nu}\left[\bar{g}_{\nu}, \bar{\phi}_{\nu}\right]$ where $\bar{g}_{\nu}$ and $\bar{\phi}_{\nu}: X \rightarrow \mathscr{K}$ are $C^{r}$ with $\bar{g}_{\nu}\left(x_{0}\right)=0$. We can easily arrange that $\bar{g}_{\nu}$ and $\bar{\phi}_{v}$ have support in $U$. Then let $g_{v}, \phi_{\nu}$ be the corresponding functions on $\mathscr{P}$. Then if $p \in \mathscr{P}$ with $\pi(p)=x$ we have,

$$
\begin{aligned}
h(p) & =\operatorname{Ad}\left(\theta(p)^{-1}\right) \bar{h}(x)=\operatorname{Ad}\left(\theta(p)^{-1}\right)\left(\sum_{\nu}\left[\bar{g}_{\nu}(x), \bar{\phi}_{\nu}(x)\right]\right) \\
& =\sum_{\nu}\left[\operatorname{Ad}\left(\theta(p)^{-1}\right) \bar{g}_{\nu}(x), \operatorname{Ad}\left(\theta(p)^{-1}\right) \bar{\phi}_{\nu}(x)\right] \\
& =\sum_{\nu}\left[g_{\nu}(p), \phi_{\nu}(p)\right]=\left(\sum_{\nu}\left[g_{\nu}, \phi_{\nu}\right]\right)(p) .
\end{aligned}
$$

2. A classification theorem. In this section, in addition to the assumptions made in the introduction, we assume $K$ is a simple Lie group with trivial center. We first make some observations.

Given a principal $K$-bundle $\pi: \mathscr{P} \rightarrow X$ we construct the associated fiber bundle $\mathscr{A} \rightarrow X$ with fiber $\mathscr{K}$ where $K$ acts on $\mathscr{K}$ via the adjoint representation of $K$. Each $p \in \mathscr{P}$ with $\pi(p)=x$ gives a linear isomorphism $\phi_{p}: \mathscr{K} \rightarrow \mathscr{A}_{x}$. Since $A d: K \rightarrow \operatorname{Lis}(\mathscr{K})$ actually takes values in $\operatorname{Aut}(\mathscr{K})$ we see $\mathscr{A}$ is a bundle of Lie algebras. Therefore $\Gamma^{r}(\mathscr{A})$, the space of $C^{r}$-sections of $\mathscr{A}, \quad$ is a Lie algebra with pointwise bracket. There is a natural isomorphism $\mathscr{H} \rightarrow \Gamma^{r}(\mathscr{A})$ given by $h \rightarrow \tilde{h}$ where $\tilde{h}(x)=\phi_{p}(h(p))$ for each $x \in X$ where $p \in \pi^{-1}(x)$ [3]. This isomorphism is an isomorphism of $\mathscr{F}(X)$-modules and is a homeomorphism with respect to the $C^{r}$-topologies.

Now suppose $\pi_{i}: \mathscr{P}_{i} \rightarrow X$ are principal $K$-bundles, $i=1,2$, with gauge groups $H_{i}$ and $\mathscr{H}_{i}$ the Lie algebra of $H_{i}$. For $x_{0} \in X$ the ideal $\mathscr{H}_{i_{0}}$ 
is closed. Let $\psi: H_{1} \rightarrow H_{2}$ be a $C^{1}$-group isomorphism. There is an induced Lie algebra isomorphism $\psi_{*}: \mathscr{H}_{1} \rightarrow \mathscr{H}_{2}$ given by

$$
\psi_{*}(h)(p)=\left.\frac{d}{d t}\right|_{t=0}[\psi(\exp (t h))](p)
$$

$\psi_{*}$ is a topological isomorphism and so for each $x_{0} \in X \psi_{*}\left(\mathscr{H}_{1 x_{0}}\right)$ is a closed ideal having property $s$ in $\mathscr{H}_{2}$. If we write $\mathscr{I}=\psi_{*}\left(\mathscr{H}_{1 x_{0}}\right)$ and refer to the discussion of section 1 we have ideals $\mathscr{K}_{x} \subset \mathscr{K}$ for each $x \in$ $X$. There are apparently two possible cases.

Case 1. $\mathscr{K}_{x}=\mathscr{K}$ for all $x \in X$.

We argue this cannot occur. Since $\mathscr{I}$ is an ideal with property $s \mathscr{I}$ is an $\mathscr{F}(X)$-submodule. If $\mathscr{K}_{x}=\mathscr{K}$ for all $x$ in $X$ we shall show $\mathscr{I}=\mathscr{H}_{2}$ which is impossible since $\mathscr{H}_{1 x_{0}} \neq \mathscr{H}_{1}$. To show $\mathscr{I}=\mathscr{H}_{2}$ we regard $\mathscr{I}$ as a closed $\mathscr{F}(X)$-submodule of $\Gamma^{r}\left(\mathscr{A}_{2}\right)$. Then for $x \in X, v \in \mathscr{A}_{2 x}$ there is $h \in \mathscr{I}$ for which $h(x)=v$. One now uses the $\mathscr{F}(X)$-module structure to show for any $x \in X$ and for any $r$-jet $\xi \in j_{x}^{r} \mathscr{A}_{2}$ there is an $h \in \mathscr{I}$ for which $j_{x}^{r} h=\xi$. Since $\mathscr{I}$ is a closed submodule we conclude $\mathscr{I}=\Gamma^{r}\left(\mathscr{A}_{2}\right)$ by applying a "global" version of a well-known theorem of Whitney. We refer to [5], Corollary 1.6, p. 25.

\section{Case 2. $\mathscr{K}_{x}=\mathscr{K}$ for some $x$.}

In this case there is some $x_{1}$ for which $\mathscr{K}_{x_{1}}=(0)$ since $K$ is simple. We claim there cannot be an $x_{2} \neq x_{1}$, for which $\mathscr{K}_{x_{2}}=0$. For if there were then we would have $\mathscr{I} \subset \mathscr{H}_{2 x_{1}} \cap \mathscr{H}_{2 x_{2}}$. But the codimension of $\mathscr{I}$ in $\mathscr{H}_{2}$ equals the codimension of $\mathscr{H}_{1 x_{0}}$ in $\mathscr{H}_{1}$ which equals the codimension of $\mathscr{H}_{2 x_{1}}$ in $\mathscr{H}_{2}$ so $\mathscr{I} \subset \mathscr{H}_{2 x_{1}} \cap \mathscr{H}_{2 x_{2}}$ is not possible. Therefore in the present case we see there is a unique $x_{1} \in X$ for which $\mathscr{I}=\mathscr{H}_{2 x_{1}}$.

Thus we see that a $C^{1}$ isomorphism $\psi: H_{1} \rightarrow H_{2}$ gives rise to a bijection $\bar{\psi}: X \rightarrow X$ defined by

$$
\psi_{*}\left(\mathscr{H}_{1 x}\right)=\mathscr{H}_{2 \bar{\psi}(x)} .
$$

Now let $h \in \mathscr{H}_{1}, f \in \mathscr{F}(X)$. We have $\bar{\psi}: X \rightarrow X$ and we write $\bar{\psi}_{*}(f)=$ $f \circ \bar{\psi}^{-1}$.

LEMMA 2.1. $\psi_{*}(f h)=\bar{\psi}_{*}(f) \psi_{*}(h)$.

Proof. Let $p_{2} \in \mathscr{P}_{2 x}$ let $\lambda=\bar{\psi}_{*}(f)(x)$. Then

$$
\begin{aligned}
\psi_{*}(f h)\left(p_{2}\right) & =\psi_{*}(f h-\lambda h)\left(p_{2}\right)+\psi_{*}(\lambda h)\left(p_{2}\right) \\
& =\psi_{*}((f-\lambda) h)\left(p_{2}\right)+\lambda \psi_{*}(h)\left(p_{2}\right) .
\end{aligned}
$$


Let $x^{\prime}=\bar{\psi}^{-1}(x)$ and let $p_{1} \in \mathscr{P}_{1 x^{\prime}}$. Then $(f-\lambda) h\left(p_{1}\right)=\left(f\left(x^{\prime}\right)-\lambda\right) h\left(p_{1}\right)=$ 0 by choice of $\lambda$. Thus $(f-\lambda) h \in \mathscr{H}_{1 x^{\prime}}$ and so $\psi_{*}((f-\lambda) h) \in \mathscr{H}_{2 x}$ so $\psi_{*}((f-\lambda) h)\left(p_{2}\right)=0$. Thus

$$
\psi_{*}(f h)\left(p_{2}\right)=\lambda \psi_{*}(h)\left(p_{2}\right)=\left(\bar{\psi}_{*}(f) \cdot \psi_{*}(h)\right)\left(p_{2}\right)
$$

as desired.

Lemma 2.2. The map $\bar{\psi}: X \rightarrow X$ is a $C^{r}$-diffeomorphism.

Proof. We need only show $\bar{\psi}^{-1}$ is $C^{r}$. It is enough to show that if $f \in \mathscr{F}(X)$ then $f \circ \bar{\psi}^{-1}$ is $C^{r}$. Choose $x_{0} \in X, U$ a neighborhood of $x_{0} \mathscr{P}_{2}$ trivial over $U$. Then let $V$ be a neighborhood of $x_{0}$ with $\bar{V} \subset U$. Let $k$ be a section of $\mathscr{A}_{2}$ over $U$ which in the local trivialization has constant principal part. We can then cut $k$ down to get a new section, again called $k$, defined on all of $X$ and agreeing with the original $k$ on $V$. Then choose $h \in \Gamma^{r}\left(\mathscr{A}_{1}\right)$ such that $\psi_{*}(h)=k$. (We are identifying $\mathscr{H}_{i}$ and $\left.\Gamma\left(\mathscr{A}_{i}\right)\right)$. Now by Lemma we have $\psi_{*}(f h)=\left(f \circ \bar{\psi}^{-1}\right) \psi_{*}(h)=$ $\left(f \circ \bar{\psi}^{-1}\right) k$. When we view the $C^{r}$-section $\left(f \circ \bar{\psi}^{-1}\right) k$ in our local trivialization we conclude $f \circ \bar{\psi}^{-1}$ is $C^{r}$ on $V$. So we conclude $f \circ \bar{\psi}^{-1}$ is $C^{r}$ and hence $\bar{\psi}^{-1}$ is $C^{r}$.

We now define a bundle isomorphism $\tilde{\psi}$. such that the following commutes:

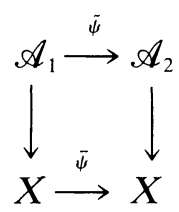

Let $\alpha_{x} \in \mathscr{A}_{1 x}$. Choose a section $h \in \Gamma^{r}\left(\mathscr{A}_{1}\right)$ such that $h(x)=\alpha_{x}$. Define $\tilde{\psi}\left(\alpha_{x}\right)$ by $\tilde{\psi}\left(\alpha_{x}\right)=\psi_{*}(h)(\bar{\psi}(x))$. This is independent of the choice of $h$ for if $h_{1}$ were another section with $h_{1}(x)=\alpha_{x}$ then $h-h_{1}$ vanishes at $x$. Hence $\psi_{*}\left(h-h_{1}\right)$ vanishes at $\bar{\psi}(x)$ so $\psi_{*}(h)(\bar{\psi}(x))=$ $\psi_{*}\left(h_{1}\right)(\bar{\psi}(x))$. It is clear that the diagram commutes and that $\tilde{\psi}$ mapping $\mathscr{A}_{1 x}$ to $\mathscr{A}_{2 \bar{\psi}(x)}$ is a Lie algebra isomorphism.

Lemma 2.3. $\tilde{\psi}$ is $C^{r}$.

Proof. We work locally trivializing $\mathscr{A}_{1}$. Let $U$ be open in $X$, $V \subset U$ also open, $\gamma: U \times R^{m} \rightarrow \mathscr{A}_{1} \mid U$ be a trivialization of $\mathscr{A}_{1}$ over $U$. Using this we see there are $C^{r}$-sections $h_{1}, \cdots, h_{m} \in \Gamma^{r}\left(\mathscr{A}_{1}\right)$ such that for each $x$ in the subset $V, h_{1}(x), \cdots h_{m}(x)$ give a basis for the fiber over $x$ which corresponds to the standard basis of $R^{m}$ under $\gamma$. We claim 
$\tilde{\psi} \circ \gamma: V \times R^{m} \rightarrow \mathscr{A}_{2}$ is given by

$$
\tilde{\psi} \circ \gamma\left(x, \xi^{1}, \cdots, \xi^{m}\right)=\sum_{i=1}^{m} \xi^{i} \psi_{*}\left(h_{\imath}\right)(\bar{\psi}(x)) .
$$

If so then $\tilde{\psi}$ is $C^{r}$. But given $\xi^{1}, \cdots, \xi^{m}$ choose $f^{\prime} \in \mathscr{F}(X)$, $f^{\prime}(x)=\xi^{\prime}$. Then by Lemma 2.1 we see

$$
\begin{aligned}
\tilde{\psi}\left(\gamma\left(x, \xi^{1}, \cdots, \xi^{m}\right)\right) & =\tilde{\psi}\left(\sum_{i=1}^{m} \xi^{i} h_{i}(x)\right)=\tilde{\psi}\left(\left(\sum_{i=1}^{m} f^{\prime} h_{\imath}\right)(x)\right) \\
& =\psi_{*}\left(\sum_{i=1}^{m} f^{\imath} h_{i}\right)(\bar{\psi}(x)) \\
& =\sum_{i=1}^{m} \bar{\psi}_{*}\left(f^{\imath}\right)(\bar{\psi}(x)) \psi_{*}\left(h_{i}\right)(\bar{\psi}(x)) \\
& =\sum_{i=1}^{m} \xi^{\prime} \psi_{*}\left(h_{\imath}\right)(\bar{\psi}(x)) .
\end{aligned}
$$

Let $p \in \mathscr{P}_{1 x}$. Then $\phi_{p}^{1}: \mathscr{K} \rightarrow \mathscr{A}_{1 x}$ is a Lie algebra isomorphism. If $q \in \mathscr{A}_{2 \bar{\psi}(x)}$ then we have a Lie algebra isomorphism $\phi_{q}^{2}: \mathscr{K} \rightarrow \mathscr{A}_{2 \bar{\psi}(x)}$. (Note the superscripts tell which bundle is being used).

Now $\left(\phi_{q}^{2}\right)^{-1} \circ \tilde{\psi} \circ \phi_{p}^{1}: \mathscr{K} \rightarrow \mathscr{K}$ lies in Aut $(\mathscr{K})$. Let $\mathscr{E}=\left\{(p, q) \mid p \in \mathscr{P}_{1 x}\right.$ and $q \in \mathscr{P}_{2 \bar{\psi}(x)}$ for some $\left.x \in X\right\} . \quad \mathscr{E}$ is the total space of the fiber product of $\mathscr{P}_{1}$ and $\bar{\psi}^{*} \mathscr{P}_{2}$. We have a map $\rho: \mathscr{E} \rightarrow \operatorname{Aut}(\mathscr{K}), \rho(p, q)=$ $\left(\phi_{q}^{2}\right)^{-1} \circ \tilde{\psi} \circ \phi_{p}^{1} . \quad \rho$ is continuous and $\mathscr{E}$ is connected so $\rho$ takes values in one of the connected components of Aut $(\mathscr{K})$. Since $K$ is a simple group the identity component of $\operatorname{Aut}(\mathscr{K})$ is $\operatorname{Aut}^{\circ}(\mathscr{K})=A d(K)$. Suppose $\sigma \in \operatorname{Aut}(\mathscr{K})$ and that $\rho(E) \subset \operatorname{Aut}^{\circ}(\mathscr{K}) \sigma=A d(K) \sigma$. Let $q \in \mathscr{P}_{2}$, $k \in K$. Then $\phi_{q k}^{2}=\phi_{q}^{2} \circ A d(k)$. So $\rho(p, q k)=A d\left(k^{-1}\right) \circ \rho(p, q)$. We conclude that for each $p \in \mathscr{P}_{1 x}$ there is a unique $\mu(p)$ in $\mathscr{P}_{2 \bar{\psi}(x)}$ for which $\rho(p, \mu(p))=\sigma$. We then have a map $\mu: \mathscr{P}_{1} \rightarrow \mathscr{P}_{2}$ covering $\bar{\psi} . \quad K$ acts freely on the right of both $\mathscr{P}_{1}$ and $\mathscr{P}_{2}$. We now show there is an automorphism $\bar{\sigma}$ of $K$, induced by $\sigma$, such that if a new action of $K$ on $\mathscr{P}_{2}$ is defined by $q * k=q \bar{\sigma}(k)$, (the right side being the original action) then $\mu$ becomes $K$-equivariant. We have $\sigma \in \operatorname{Aut}(\mathscr{K}) . \quad \tau \rightarrow \sigma \tau \sigma^{-1}$ is an automorphism of Aut $(\mathscr{K})$ and hence restricts to an automorphism of $\operatorname{Aut}^{\circ}(\mathscr{K})=A d(K)$. Using the isomorphism $A d: K \rightarrow A d(K)$ we see a unique automorphism $\bar{\sigma}$ is induced. $\bar{\sigma}$ satisfies the equation $A d(\bar{\sigma}(k))=\sigma A d(k) \sigma^{-1}$. Now we show $\mu(p k)=\mu(p) * k$ for $p \in \mathscr{P}_{1}$, $k \in K$. We need only show $\rho(p k, \mu(p) * k)=\sigma$. But

$$
\begin{aligned}
\rho(p k, \mu(p) * k) & =\rho(p k, \mu(p) \bar{\sigma}(k))=A d(\bar{\sigma}(k))^{-1} \circ \rho(p, \mu(p)) \circ \operatorname{Ad}(k) \\
& =\operatorname{Ad}(\bar{\sigma}(k))^{-1} \circ \sigma \circ \operatorname{Ad}(k)=\sigma A d(k)^{-1} \sigma^{-1} \sigma A d(k)=\sigma
\end{aligned}
$$

so we are done. 
Definition 2.4. Let $\pi: \mathscr{P} \rightarrow X$ be a principal $K$-bundle, $\tau$ an automorphism of $K$. The principal $K$-bundle $\pi^{\tau}: \mathscr{P}^{\tau} \rightarrow X$ is defined by introducing the new action $*: \mathscr{P} \times K \rightarrow P, p * k=p \tau(k)$. We say $\pi^{\tau}$ is conjugate to $\pi$ by $\tau$.

Considering the previous discussion we have now proved

THEOREM 2.5. Under the assumptions made above if $\psi: H_{1} \rightarrow H_{2}$ is a $C^{1}$ isomorphism then there is a $C^{r}$-diffeomorphism $\bar{\psi}: X \rightarrow X$ and an automorphism $\bar{\sigma}$ of $K$ such that $\pi_{1} \cong \bar{\psi}^{*}\left(\pi_{2}^{\bar{\sigma}}\right)$.

REMARK. Of course if $\bar{\sigma}$ is an inner automorphism we get $\pi_{2}^{\bar{\sigma}} \cong \pi_{2}$ and $\bar{\sigma}$ can be dropped.

3. Classical groups. We apply the results of $\S 2$ to the groups $\mathrm{SO}(2 n+1) n \geqq 1, U(n) n \geqq 2$, and SO $(2 n) n \geqq 3$. Since the center of $\mathrm{SO}(2 n+1)$ is trivial and the automorphism group of its Lie algebra is connected [6, pages 285-6] we get

Theorem 3.1. Let $\pi_{i}: \mathscr{P}_{i} \rightarrow X$ be principal $\mathrm{SO}(2 n+1)$ bundles with gauge groups $H_{i}, i=1,2$. Suppose $\psi: H_{1} \rightarrow H_{2}$ is a $C^{1}$ (local) isomorphism. Then there is a $C^{r}$-diffeomorphism $\bar{\psi}: X \rightarrow X$ so that $\pi_{1} \cong \bar{\psi}^{*}\left(\pi_{2}\right)$.

Now let $K$ be $\mathrm{SO}(2 n) n \geqq 3$ or $U(n) n \geqq 2, \pi_{i}: \mathscr{P}_{i} \rightarrow X$ be principal $K$ bundles with gauge groups $H_{t}$ and $\psi: H_{1} \rightarrow H_{2}$ a $C^{r}$ local isomorphism. Let $Z$ denote the center of $K$. Now $\hat{\mathscr{P}}_{l}=\mathscr{P}_{\imath} / Z$ is a principal $K / Z$ bundle over $X$. Let $\hat{H}_{i}$ be the gauge group of $\hat{\mathscr{P}}_{1}$. In both cases $(\mathrm{SO}(2 n)$ and $U(n))$ one can show that the Lie algebra isomorphism $\psi_{*}: \mathscr{H}_{1} \rightarrow \mathscr{H}_{2}$ gives Lie algebra isomorphism $\hat{\psi}_{*}: \hat{\mathscr{H}}_{1} \rightarrow \hat{\mathscr{H}}_{2}$ and also that the center of $K / Z$ is trivial. Thus the results of $\S 2$ give a $C^{r}$ diffeomorphism $\phi: X \rightarrow X$ and an automorphism $\sigma$ of $K / Z$ so that $\hat{\pi}_{1} \cong \phi^{*}\left(\hat{\pi}_{2}^{\sigma}\right)$. Note that if $\sigma$ is an inner automorphism $\hat{\pi}_{2}^{\sigma} \cong \hat{\pi}_{2}$ so that $\sigma$ can be dropped. The form of $\sigma$ not inner is given in [6, page 287]. It can be seen that $\sigma$ lifts to $\sigma: K \rightarrow K$ and that $\left(\mathscr{P}_{l} / Z\right)^{\sigma}=\mathscr{P}_{l}^{\sigma} / Z$. We thus get

Theorem 3.2. Let $K$ be $\mathrm{SO}(2 n) n \geqq 3$ or $U(n) n \geqq 2, \pi_{i}: \mathscr{P}_{\imath} \rightarrow X$ be principal $K$ bundles with gauge groups $H_{i}, i=1,2$. Suppose $\psi: H_{1} \rightarrow H_{2}$ is a (local) $C^{r}$ isomorphism. Then there is a $C^{r}$ diffeomorphism $\bar{\psi}: X \rightarrow X$ and automorphism $\sigma: K \rightarrow K$, so that $\mathscr{P}_{1} / Z \cong$ $\bar{\psi}^{*}\left(\mathscr{P}_{2} \mid Z\right)^{\sigma} \cong \bar{\psi}^{*}\left(\mathscr{P}_{2}^{\sigma}\right) / Z$ where $Z$ is the center of $K$.

One can show that $\mathscr{P}_{1}$ is a "tensor product" of $\bar{\psi}^{*}\left(\mathscr{P}_{2}^{\sigma}\right)$ with a 
principal $Z$-bundle over $X$. One way to see this is to use the classification for bundles as given in [4]. We state the result in terms of associated vector bundles.

THEOREM 3.3. Let $\pi_{i}: \mathscr{P}_{i} \rightarrow X$ be principal $\mathrm{SO}(2 n) n \geqq 3(U(n)$ $n \geqq 2$ ) bundles with gauge groups $H_{i}, i=1,2$. Let $\xi_{i}$ be the real (complex) vector bundle associated with $\mathscr{P}_{i}$ using the usual representation of SO $(2 n)(U(n))$. Suppose $\psi: H_{1} \rightarrow H_{2}$ is a (local) $C^{1}$-isomorphism then there is a $C^{r}$ diffeomorphism $\bar{\psi}: X \rightarrow X, \sigma$ an automorphism of $\mathrm{SO}(2 n)(U(n))$, and $\eta$ a real (complex) line bundle so that $\xi_{1}$ is $\mathrm{SO}(2 n)(U(n))$ isomorphic to $\psi^{*}\left(\xi_{2}^{\sigma}\right) \otimes \eta$.

Final remark. We need not have assumed that $\mathscr{P}_{1}$ and $\mathscr{P}_{2}$ were bundles over the same manifold $X$. We could have considered $\pi_{1}: \mathscr{P}_{1} \rightarrow X$ and $\pi_{2}: \mathscr{P}_{2} \rightarrow Y$. If the gauge groups $H_{1}$ and $H_{2}$ are (locally) $C^{1}$ isomorphic we get a $C^{r}$-diffeomorphism $\bar{\psi}: X \rightarrow Y$.

\section{REFERENCES}

1. W. D. Curtis, The automorphism group of a compact group action, Trans. Amer. Math. Soc., 203 (1975), 45-54.

2. W. D. Curtis, Y-L. Lee and F. R. Miller, A class of infinite dimensional subgroups of Diff ${ }^{\prime}(x)$ which are Banach Lie groups, Pacific J. Math., 47 (1973), 59-65.

3. Kobayashi and Nomizu, Foundations of Differential Geometry Vol. I, Interscience, New York 1963.

4. R. Lashof, Classification of Fibre bundles by the loop space of the base, Annals of Math., 64 (1965), 436-446.

5. B. Malgrange, Ideals of Differentiable Functions, Oxford Univ. Press 1966.

6. J. A. Wolf, Spaces of Constant Curvature, Third Edition, Publish or Perish Inc., Boston Mass., 1974.

Received June 8, 1976.

Kansas State University

MANHATTAN, KS 66506 


\section{PACIFIC JOURNAL OF MATHEMATICS}

\section{EDITORS}

RichaRd ARENS (Managing Editor)

University of California

Los Angeles, CA 90024

\section{R. A. Beaumont}

University of Washington

Seattle, WA 98105

C. C. Moore

University of California

Berkeley, CA 94720
J. DugunduI

Department of Mathematics

University of Southern California

Los Angeles, CA 90007

R. Finn AND J. Milgram

Stanford University

Stanford, CA 94305

\section{ASSOCIATE EDITORS}
E. F. BECKENBACH
B. H. NeumanN
F. WOLF
K. YoshidA

\section{SUPPORTING INSTITUTIONS}

UNIVERSITY OF BRITISH COLUMBIA

CALIFORNIA INSTITUTE OF TECHNOLOGY

UNIVERSITY OF CALIFORNIA

MONTANA STATE UNIVERSITY

UNIVERSITY OF NEVADA

NEW MEXICO STATE UNIVERSITY

OREGON STATE UNIVERSITY

UNIVERSITY OF OREGON

OSAKA UNIVERSITY

\author{
UNIVERSITY OF SOUTHERN CALIFORNIA \\ STANFORD UNIVERSITY \\ UNIVERSITY OF HAWAII \\ UNIVERSITY OF TOKYO \\ UNIVERSITY OF UTAH \\ WASHINGTON STATE UNIVERSITY \\ UNIVERSITY OF WASHINGTON \\ AMERICAN MATHEMATICAL SOCIETY
}

The Supporting Institutions listed above contribute to the cost of publication of this Journal, but they are not owners or publishers and have no responsibility for its contents or policies.

Mathematical papers intended for publication in the Pacific Journal of Mathematics should be in typed form or offset-reproduced (not dittoed), double spaced with large margins. Underline Greek letters in red, German in green, and script in blue. The first paragraph or two must be capable of being used separately as a synopsis of the entire paper. Items of the bibliography should not be cited there unless absolutely necessary, in which case they must be identified by author and Journal, rather than by item number. Manuscripts, in duplicate, may be sent to any one of the four editors. Please classify according to the scheme of Math. Reviews, Index to Vol. 39. All other communications should be addressed to the managing editor, or Elaine Barth, University of California, Los Angeles, California, 90024.

100 reprints are provided free for each article, only if page charges have been substantially paid. Additional copies may be obtained at cost in multiples of 50 .

The Pacific Journal of Mathematics is issued monthly as of January 1966. Regular subscription rate: $\$ 72.00$ a year (6 Vols., 12 issues). Special rate: $\$ 36.00$ a year to individual members of supporting institutions.

Subscriptions, orders for back numbers, and changes of address should be sent to Pacific Journal of Mathematics, 103 Highland Boulevard, Berkeley, California, 94708.

PUBLISHED BY PACIFIC JOURNAL OF MATHEMATICS, A NON-PROFIT CORPORATION

Printed at Jerusalem Academic Press, POB 2390, Jerusalem, Israel. 


\section{Pacific Journal of Mathematics}

Vol. 68, No. 2

April, 1977

William Allen Adkins, Aldo Andreotti and John Vincent Leahy, An analogue of Oka's theorem for weakly normal complex spaces ........

Ann K. Boyle, M. G. Deshpande and Edmund H. Feller, On nonsingularly

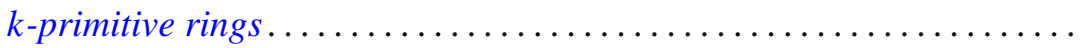

Rolando Basim Chuaqui, Measures invariant under a group of

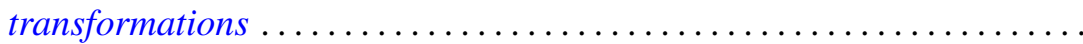

Wendell Dan Curtis and Forrest Miller, Gauge groups and classification of bundles with simple structural group .......................

Garret J. Etgen and Willie Taylor, The essential uniqueness of bounded nonoscillatory solutions of certain even order differential equations

Paul Ezust, On a representation theory for ideal systems

Richard Carl Gilbert, The deficiency index of a third order operator ........

John Norman Ginsburg, $S$-spaces in countably compact spaces using Ostaszewski's method.

Basil Gordon and S. P. Mohanty, On a theorem of Delaunay and some

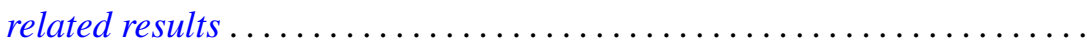

Douglas Lloyd Grant, Topological groups which satisfy an open mapping

theorem.

Charles Lemuel Hagopian, A characterization of solenoids

Kyong Taik Hahn, On completeness of the Bergman metric and its

subordinate metrics. II .

G. Hochschild and David Wheeler Wigner, Abstractly split group extensions.

Gary S. Itzkowitz, Inner invariant subspaces ...............

Jiang Luh and Mohan S. Putcha, A commutativity theorem for

non-associative algebras over a principal ideal domain.

Donald J. Newman and A. R. Reddy, Addendum to: "Rational approximation of $e^{-x}$ on the positive real axis".....

Akio Osada, On the distribution of a-points of a strongly annular function ....

Jeffrey Lynn Spielman, A characterization of the Gaussian distribution in a Hilbert space. .

Robert Moffatt Stephenson Jr., Symmetrizable-closed spaces ...

Peter George Trotter and Takayuki Tamura, Completely semisimple inverse $\Delta$-semigroups admitting principal series . . . . . . . .

Charles Irvin Vinsonhaler and William Jennings Wickless, Torsion free abelian groups quasi-projective over their endomorphism rings...

Frank Arvey Wattenberg, Topologies on the set of closed subsets ... 